\title{
Anomalous Entry Flow Patterns in the Transition Regime to Global Flow Instability Generated after Vortex Enhancement
}

\author{
Kunji CHIBA ${ }^{1, *}$, Itaru YoshIDA ${ }^{2}$, Satoshi $\mathrm{SAKO}^{2}$, and Noriyasu MORI ${ }^{2}$ \\ ${ }^{1}$ Faculty of Education, Shiga University, 2-5-1 Hiratsu, Otsu, Shiga 520-0862, Japan \\ ${ }^{2}$ Department of Mechanophysics Engineering, Graduate School of Engineering, \\ Osaka University, 2-1 Yamada-Oka, Suita, Osaka 565-0871, Japan
}

(Received : June 14, 2004)

\begin{abstract}
This study is concerned with an origin of entry flow instability generated after vortex enhancement in elastic fluid flows. For this purpose flow visualization experiments of shear-thinning aqueous polymer solution were carried out by means of both tracer-particle method and dye-solution injection technique. Local instability in the salient corner vortex, which was observed as non-smooth particle paths, could evolve to globally unstable entry flow as the Weissenberg number increased. The visualization experiments using the dye-solution injection technique demonstrated that the main cause of the local instability was the occurrence and decay of a dip of the vortex boundary at the lip corner and the small amplitude oscillatory phenomenon of the detachment point had a secondary influence. It was, therefore, concluded that the occurrence of the dip at the lip corner was a trigger of the global entry flow instability after vortex enhancement. However, the mechanism of the occurrence of the dip at the lip corner remains to be an open question, thus it will be necessary to measure the transient velocity field near the lip corner using the LDV or PIV technique.
\end{abstract}

Key Words: Local instability/Circular entry flow/Vortex enhancement/Flow visualization/Dye-solution injection technique

\section{INTRODUCTION}

In two decades much effort has been expended on flow instabilities, since flow instabilities occur in a variety of commercially important polymer processing operations, such as fiber spinning, film blowing, extrusion and so on, and they also seriously affect the production rate and quality of products.

One of the well-known research works concerning instability of entry flow of polymer solutions is the visualization of $4: 1$ circular entry flow of polyacrylamide solutions conducted by Cable and Boger. ${ }^{1)}$ They observed spiraling, pulsing and random flow distortions as the flow rate is increased. Nguyen and Boger $^{2}$ observed periodic helical flow in a 7.67:1 circular contraction for a Boger fluid: flow lines moved into the contraction like a tornado, then a large new vortex was formed and the process was repeated. Cartalos and Piau ${ }^{3)}$ conducted flow visualizations of 20 500 ppm solutions of partially hydrolyzed polyacrylamide through a $16: 1$ orifice die, and reported that a 'turning flower' flow and 'knitting' flow were first observed. Furthermore, shorter exposure time photographs revealed small-scale instabilities throughout a converging

* Corresponding author

Tel : 077-537-7800, Fax: 077-537-7840

E-mail: kchiba@sue.shiga-u.ac.jp zone. The same kind of instabilities was observed by Piau et al. ${ }^{4}$ for linear and branched silicone fluids.

Furthermore, concerning the local instability of entry flow, small amplitude periodic fluctuations are clearly visible in numerous enlarged photographs in the flow visualization book of Boger and Walters. ${ }^{5)} \mathrm{Naka}^{6}$ also carried out flow visualizations of $0.2 \mathrm{wt} \%$ and $0.5 \mathrm{wt} \%$ polyacrylamide aqueous solutions through a 4:1 circular contraction, and observed the local flow instability in the salient corner vortex prior to the onset of global instability. Figure 1, which is reproduced by his permission, shows typical local flow instability. We can find non-smooth particle paths near the detachment point of the salient corner vortex in Fig. 1(b) (Weissenberg number, $W e=1.36$ ), then the non-smooth particle paths evolve toward the vortex center as We increases from Fig. 1(c) to Fig. 1(f). The entry flow at $W e=2.71$ (Fig. 1(f)) was confirmed to be not globally unstable. Furthermore, enlarged photographs taken at the different exposure times ( $70 \mathrm{~s}$ and $10 \mathrm{~s})$ are also reproduced in Fig. 2: non-smooth particle paths can be clearly seen near the detachment point (region 'A'), in the salient corner vortex (region ' $C$ ') as well as intersections of particle paths in the main flow region near the vortex boundary (region 'B'). Nonsmooth particle paths in the main flow are clearly visible in the 
short exposure time photograph (Fig. 2(b)). Naka ${ }^{6}$ concluded that the local instability was caused by the small amplitude oscillatory phenomenon of the detachment point owing to the interaction between inertial force and elastic force, and the

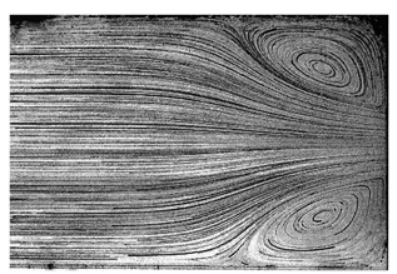

(a) $R e=0.340, W e=1.18$

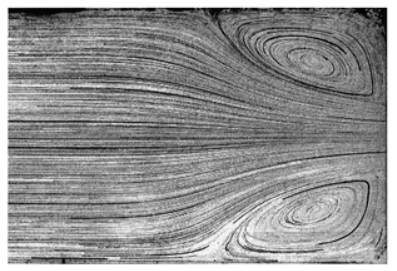

(b) $R e=0.570, W e=1.36$

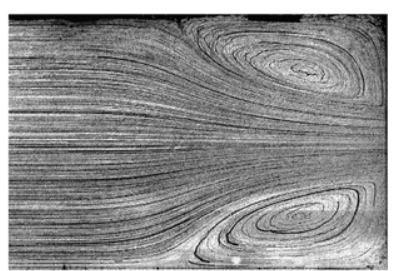

(c) $R e=1.14, W e=1.64$

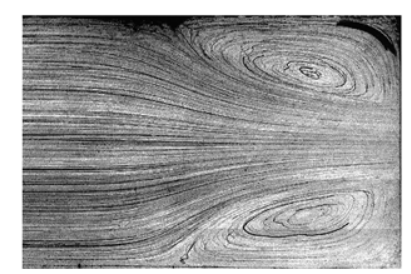

(d) $R e=1.62, W e=1.80$

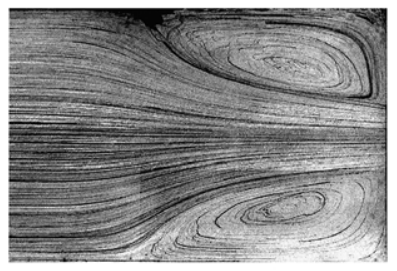

(e) $R e=2.54, W e=2.03$

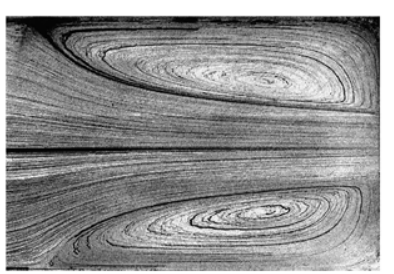

(f) $R e=7.36, W e=2.71$
Fig. 1. Typical evolution of local entry flow instability for $0.2 \mathrm{wt} \%$ polyacrylamide solution in tap water through an abrupt $4: 1$ circular contraction at $W e=1.18 \sim 2.71\left(\mathrm{Naka}^{6}\right)$.
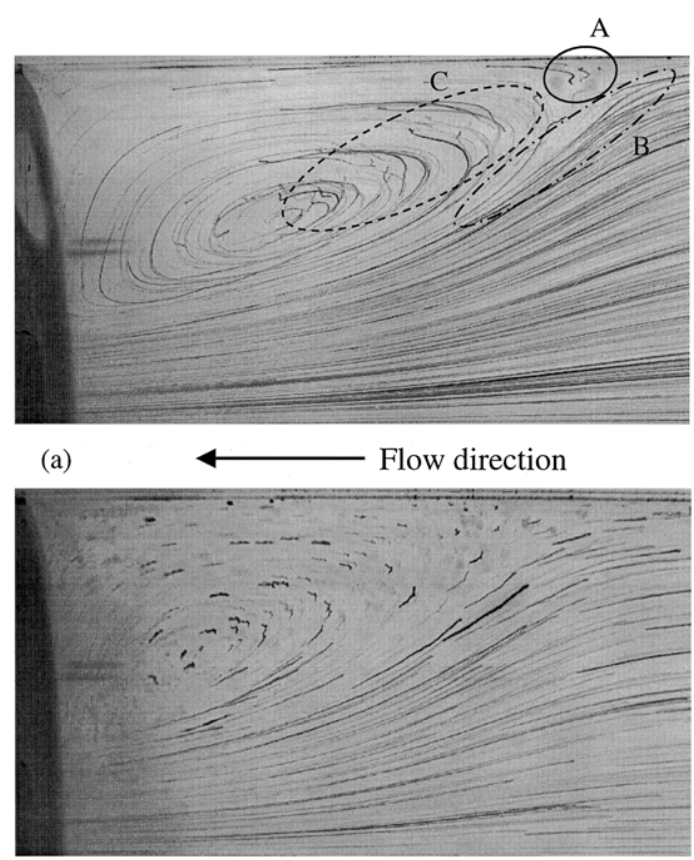

(b)

Fig. 2. Typical particle paths in the regime of local flow instability for $0.2 \mathrm{wt} \%$ polyacrylamide solution in tap water through an abrupt 4:1 circular contraction at $R e=1.65, W e=1.75 \quad\left(\mathrm{Naka}^{6}\right)$; (a) exposure time $=70 \mathrm{~s}$; (b) exposure time $=10 \mathrm{~s}$. local instability in the vortex region evolved to the global entry flow instability as the Weissenberg number was further increased.

Quantitative measurements of the fluctuations in the fluid velocity using LDV were made by Lawler et al. ${ }^{7)}$ and McKinley et al. ${ }^{8)}$ These studies clearly showed that global onset of oscillatory instability was preceded by periodic and then aperiodic fluctuations in the velocity field close to the contraction plane. However, these studies have not described in detail an origin of global instability of entry flow after salient corner vortex enhancement.

Our previous papers demonstrated further experimental evidence for entry flow instabilities of aqueous solutions of polyacrylamide through 2:1 and 4:1 circular contractions ${ }^{9), 10)}$, and also an eccentric 4:1 circular contraction. ${ }^{11)}$ For weakly elastic fluids the Goertler-like vortices occur around the salient corner owing to the reduction of the curvature radius of the main flow due to interaction between fluid elasticity and inertia, and they move back and forth along the channel wall. It has been found that the reduction of the curvature radius of the main flow is an origin of entry flow instability for weakly elastic fluids. ${ }^{9), 10)}$ It was reviewed and is well known that the elastically-driven local instability arises from the reduction of the curvature radius of the flow. ${ }^{12), 13)}$ On the other hand, the salient corner vortex grows owing to fluid elasticity, and its length is varied with time and along the circumferential direction in the global flow instability regime for rather elastic fluids. However, in this case an origin remains to be fully understood.

The objective of this study is to understand an origin of entry flow instability generated after vortex enhancement in rather elastic fluid flows on the basis of flow visualization experiments of shear-thinning aqueous polymer solutions using both tracer-particle and dye-solution injection methods.

\section{EXPERIMENTAL}

Flow visualization experiments of $0.1 \mathrm{wt} \%$ aqueous solutions of polyacrylamide (PAA; SANFLOC AH70P, $M_{w} \approx 10$ million, Sanyo Kasei Kougyo, Japan) were conducted in a circular contraction. The shear viscosity $\eta$ and the first normal stress difference $N_{1}$ of the solution was measured with a cone-and-plate rheometer at the solution temperature of $24 \sim 25^{\circ} \mathrm{C}$, and the results are shown in Fig. 3(a) and (b). It is well known that both $\eta$ and $N_{1}$ can exhibit a power-law behavior over a wide range of shear rate $\dot{\gamma}$ (see Fig. 3(a)), then the shear stress $\tau$ and $N_{1}$ may be written as

$$
\tau=K \dot{\gamma}^{n}, \quad N_{1}=A \dot{\gamma}^{m},
$$


where $n$ and $K$ are the flow index and the consistency, respectively, for the power-law model, and $m$ and $A$ are also constants. We can, furthermore, see the power law relationship between $\tau$ and $N_{1}$ from Fig. 3(b), then

$$
N_{1}=a \tau^{b}
$$

For polymeric liquids, it is often found that a plot of $\tau$ vs. $N_{1}$ for a range of temperatures results in a unique relationship and the exponent $b$ is nearly equal to $2 .{ }^{14), 15), 16)}$ The constants in Eqs.(1) and (2) are shown in Table I. In Fig. 3 and Table I the results for the $0.1 \mathrm{wt} \%$ PAA solutions in both tap water and

Table I Power-law parameters in Eqs.(1) and (2) for the $0.1 \mathrm{wt} \%$ PAA solutions at $25.2^{\circ} \mathrm{C}$ in dechlorinated tap water and $23.9^{\circ} \mathrm{C}$ in tap water.

\begin{tabular}{cccccccc}
\hline solvent & $\mathrm{K}\left(\mathrm{Pa} \cdot \mathrm{s}^{\mathrm{n}}\right)$ & $\mathrm{n}$ & $\mathrm{A}\left(\mathrm{Pa} \cdot \mathrm{s}^{\mathrm{m}}\right)$ & $\mathrm{m}$ & $\mathrm{a}\left(\mathrm{Pa} \mathrm{P}^{1-\mathrm{b}}\right)$ & $\mathrm{b}$ \\
\hline tap water & 0.126 & 0.496 & 0.0862 & 1.074 & 7.644 & 1.885 \\
dechlorinated water & 0.283 & 0.391 & 0.2909 & 0.958 & 6.801 & 2.117 \\
\hline
\end{tabular}

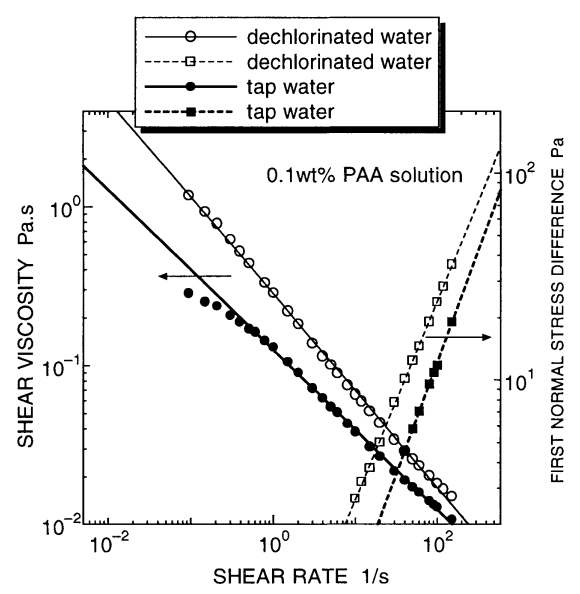

(a)

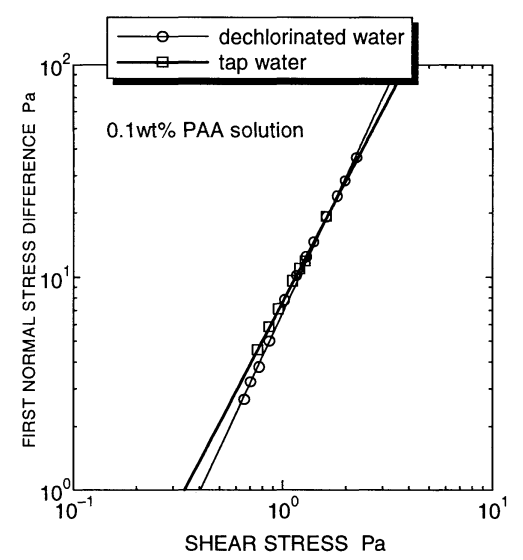

(b)

Fig. 3. Rheological properties for the $0.1 \mathrm{wt} \%$ aqueous solution of PAA, $25.2^{\circ} \mathrm{C}$ in dechlorinated tap water and $23.9^{\circ} \mathrm{C}$ in tap water; (a) Shear viscosity and the first normal stress difference as a function of shear rate; (b) The first normal stress difference vs. shear stress. dechlorinated tap water are included: $\mathrm{Naka}^{6}$, Chiba and Nakamura9), Naka et al. ${ }^{10,11)}$ used solution in tap water in the previous studies, while solution in dechlorinated tap water was used in the present study because of suppression of the solution degradation. It is clearly shown that both $\eta$ and $N_{1}$ become larger and also shear-thinning behavior is strongly exhibited for the solution in dechlorinated tap water. Although it was found that the flow properties of the two aqueous solutions are much different, the effect of dechlorinated tap water on the entry flow will not be taken into account in this paper.

Figure 4 shows the schematic diagram of the experimental setup. Constant pressure in a head tank was supplied with a compressor to keep the flow rate constant. The circular tube was surrounded by a rectangular viewing box, which was filled with a refractive-index-matching fluid to minimize optical distortion. In the flow visualization experiments, we used not only the tracer method but also the dye-solution injection technique in order to examine the flow structure and an origin of entry flow instabilities. Polystyrene spheres smaller than $100 \mu \mathrm{m}$ in diameter were used as tracers and a planar sheet (approximately $1 \mathrm{~mm}$ in thickness) of $5 \mathrm{~mW}$ He-Ne laser light source illuminated the flow in the observation plane. A planar light source was generated using a cylindrical lens. On the other hand, in the dye-solution injection visualizations a methylene blue dye solution with the same density as the polymer solution was very slowly injected through a syringe needle of $1 \mathrm{~mm}$ in diameter provided on the side wall approximately $300 \mathrm{~mm}$ upstream from the contraction. The photographic images of the particle paths and streaklines were recorded by a digital camera (Nikon D-1H).

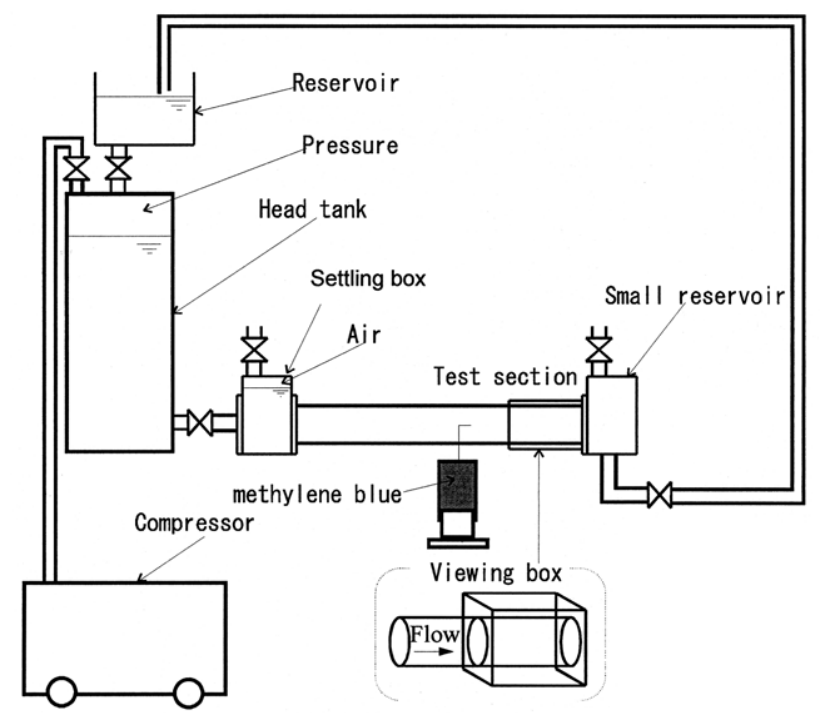

Fig. 4. Schematic diagram of the experimental apparatus. 
A schematic diagram of a circular contraction part is shown in Fig. 5: a 4:1 contraction with $40 \mathrm{~mm}$ and $10 \mathrm{~mm}$ in upstream- and downstream-tube diameters, respectively. In the tracer method, the observation planes were not only central planes (Cross section A or B) but also a plane parallel to the contraction face (Cross section $\mathrm{C}$ ) in order to examine the three-dimensional flow structure.

Finally, as the dimensionless parameters on the experimental conditions, the Reynolds number and the Weissenberg number for the flow through a contraction have been defined by

$$
\begin{aligned}
& R e=\rho U \cdot 2 R_{d} / \eta, \\
& W e=\lambda U / 2 R_{d},
\end{aligned}
$$

where $\rho$ is the fluid density, $\eta$ the shear-rate-dependent viscosity, $U$ the mean velocity in the contraction and $R_{d}$ the contraction radius. The shear-rate-dependent relaxation time is given by $\lambda=N_{1} / 2 \tau \dot{\gamma}$. The temperature correction of the rheological properties of the polymer solution was done using the method described in the previous paper. ${ }^{9)}$

\section{GENERAL VIEW FOR TRANSITION FROM STEADY TO GLOBAL INSTABILITY}

As well known, the transition state from steady to globally unstable entry flows is generated as the Weissenberg number increases. The steady entry flow shows a so-called wine-glass shaped main flow with convex boundary of the salient corner vortex and the salient corner vortex always grows with an increase in $W e$. Then non-smooth particle paths can be observed in the salient corner vortex as $W e$ is further increased

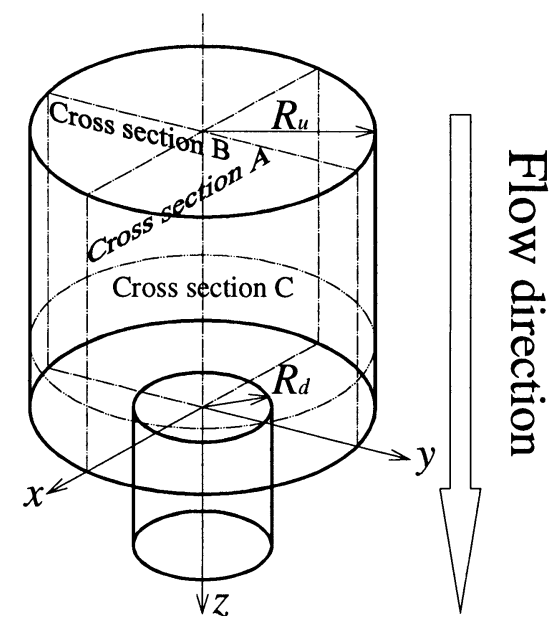

Fig. 5. Schematic diagram of a circular contraction and observation planes: Cross section A or B is the central observation plane and Cross section $\mathrm{C}$ is the observation plane parallel to the contraction face. (see photographs reproduced in Fig. 1). We also refer to this flow regime as "local flow instability" in this study. Finally, the main flow reaches the almost periodic spiraling flow and the length of the corner vortex varies along the circumferential direction, furthermore, the corner vortex rotates along the circular wall owing to the spiraling main flow, i.e., "global flow instability". Thus the alternate growth and decay of the corner vortices are seen when we observe the flow patterns in the Cross section A or B (see Fig. 5).

In order to examine the three-dimensional structure of the above three types of flow regimes, we observed the flow patterns in the plane parallel to the contraction face. Figures 6 and 7 show the particle paths near the contraction face (approximately $2 \mathrm{~mm}$ upstream location from the contraction face) and near the vortex center (the location of the vortex center depends on the flow rate), respectively. The particle paths near the contraction face become perfectly radial in the steady and local instability flow regime (Figs.6(a) (c)), in contrast, the particle paths become curved in the globally unstable flow regime (Fig. 6(d)). On the other hand, the particle paths near the vortex center align in the radial direction for steady flow and at the initial stage of local instability (Fig. 7(a) and (b)), however, they become curved for fully local instability (Fig. 7(c)), then concentric circles for global instability (Fig. 7(d)) as We is further increased. We confirmed two stages of the local instability flow regime in the salient

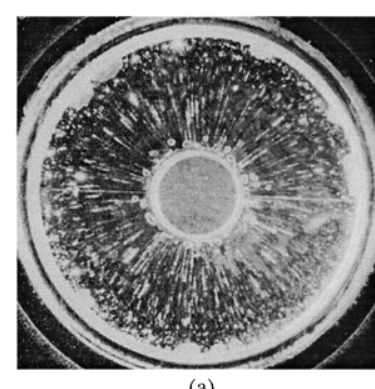

(a)

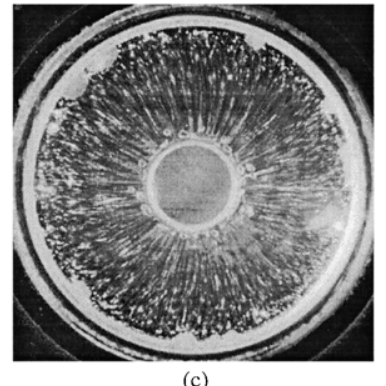

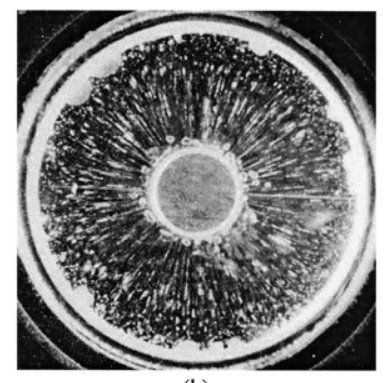

(b)

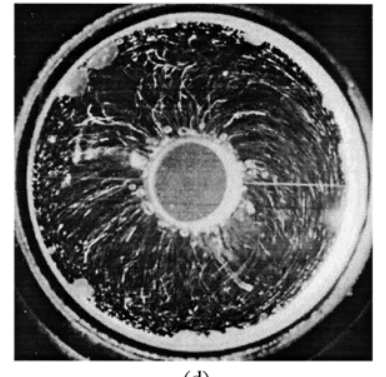

Fig. 6. Flow patterns near the contraction face for the $0.1 \mathrm{wt} \%$ aqueous solution of PAA through an abrupt 4:1 circular contraction at $25^{\circ} \mathrm{C}$; (a) $R e=0.232, W e=0.440$; (b) $R e=0.843, W e=0.625$; (c) $R e=32.7, W e=1.69$; (d) $R e=162, W e=2.60$. 
corner vortex: $W e=0.625$ (Fig. $7($ b) ) corresponds to the initial stage of the local instability and $W e=1.69$ (Fig. 7(c)) the fully local instability regime. At the initial stage of the occurrence of the local instability, therefore, the circumferential component of the fluid velocity does not exist, i.e., axisymmetric flow is generated, while the transition from axisymmetric vortex to three-dimensional vortex flow can be observed even in the local instability flow regime as $W e$ increases to approximately 1.5. We define such a flow regime as the "fully local instability". Furthermore, this three-dimensional vortex flow exhibits aperiodic change in rotation direction along the circular wall.

The tracer method is very popular in the flow visualization experiments and the particle paths can be observed, while the dye-solution injection technique has been sometimes used and it is useful and appropriate to understand the local flow patterns from the streakline. We have obtained important flow patterns in the central region of the tube which could not be detected using the tracer method. Figure 8 shows the streaklines for steady flow (a), the initial stage of the local instability (b), fully local instability (c) and globally unstable flow regime (d). In this case the dye-solution was slowly injected on the centerline. The streaklines are straight line along the centerline in Fig. 8(a) and (b), while the curved streakline can be seen clearly in the fully local instability regime in Fig. 8(c), This curved streakline seems to be caused by the occurrence of a spiraling main flow, however, we confirmed that the streakline

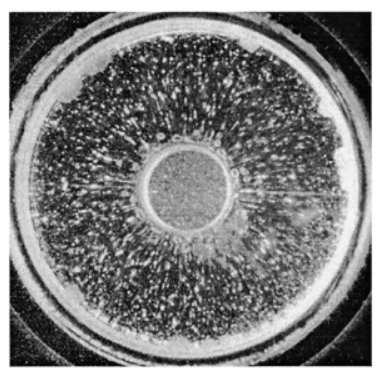

(a)

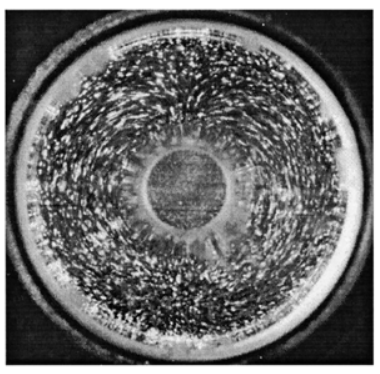

(c)

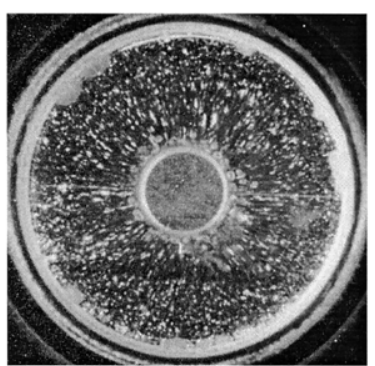

(b)

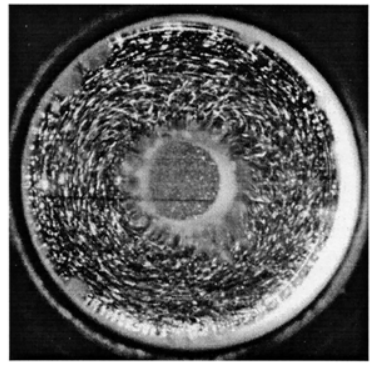

(d)
Fig. 7. Flow patterns near the vortex center for the $0.1 \mathrm{wt} \%$ aqueous solution of PAA through an abrupt 4:1 circular contraction at $25^{\circ} \mathrm{C}$; (a) $R e=0.232, W e=0.440$; (b) $R e=0.843$, $W e=0.625$; (c) $R e=32.7, W e=1.69 ;$ (d) $R e=162, W e=2.60$. was just two-dimensionally curved and spiraling main flow was not generated. This phenomenon could not be recognized in the visualizations using the tracer method. Finally, in the globally unstable flow regime the distortion of the streakline can be seen in Fig. 8(d). We can easily suppose a spiraling main flow from Fig. 11 in the next section.

\section{OCCURRENCE PROCESS OF LOCAL INSTABILITY IN THE VORTEX REGION}

The consecutive photographs were taken every $10 \sim 40 \mathrm{~s}$ in order to study the transition of vortex flow structure from local instability to globally unstable flow regimes. The streamlines in the vortex region become always smooth in the steady flow regime. As $W e$ is further increased non-smooth particle paths can be observed in the region from the detachment point to the vortex center. At the initial stage of the occurrence of local instability, both appearance and disappearance of local instability are often observed (not shown here) and also appearance of local instability is aperiodic. When We increases to 1.21, all photographs shows the local instability in Fig. 9.

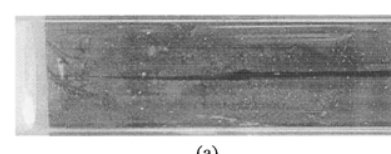

(a)
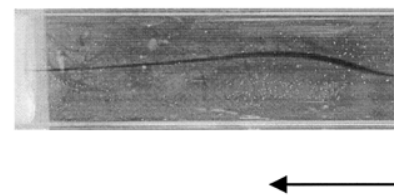

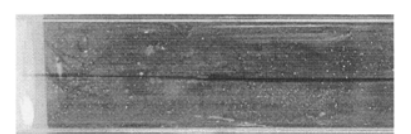

(b)

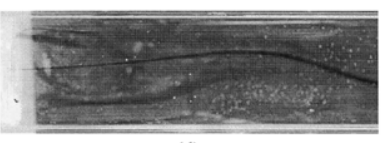

(d)
Fig. 8. Streaklines in the central region of the tube for the $0.1 \mathrm{wt} \%$ aqueous solution of PAA through an abrupt 4:1 circular contraction at $25^{\circ} \mathrm{C}$; (a) $R e=0.324$, We $=0.482$; (b) $R e=1.71$, $W e=0.757$; (c) $R e=32.7, W e=1.69$; (d) $R e=101, W e=2.28$.

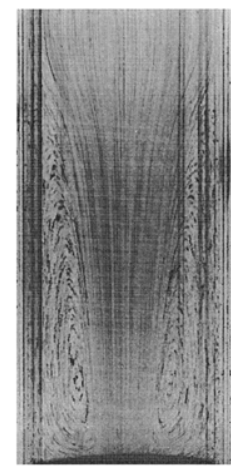

(a) $0 \mathrm{~s}$

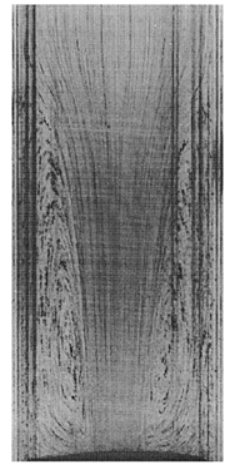

(b) $80 \mathrm{~s}$

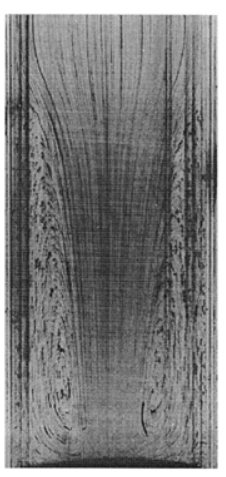

(c) $160 \mathrm{~s}$
Fig. 9. Consecutive photographs of particle paths for the $0.1 \mathrm{wt} \%$ aqueous solution of PAA through an abrupt 4:1 circular contraction at $R e=9.68, W e=1.21$ and $25^{\circ} \mathrm{C}$ in the local instability regime. 


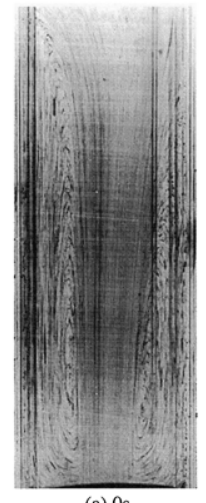

(a) $0 \mathrm{~s}$

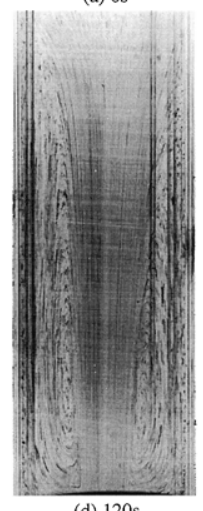

(d) $120 \mathrm{~s}$

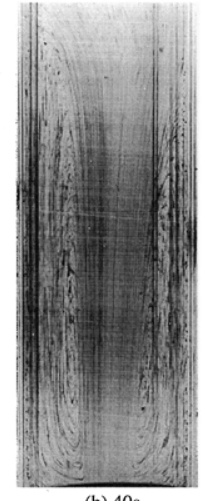

(b) $40 \mathrm{~s}$

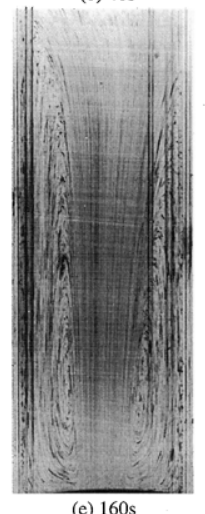

(e) $160 \mathrm{~s}$

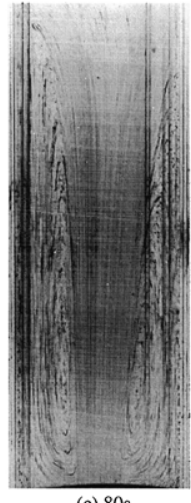

(c) $80 \mathrm{~s}$

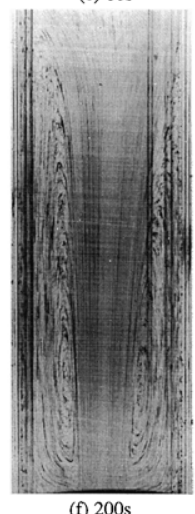

(f) $200 \mathrm{~s}$
Fig. 10. Consecutive photographs of particle paths for the $0.1 \mathrm{wt} \%$ aqueous solution of PAA through an abrupt 4:1 circular contraction at $R e=69.3, W e=2.07$ and $25^{\circ} \mathrm{C}$ in the fully local instability regime.

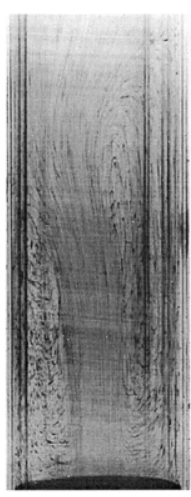

(a) $0 \mathrm{~s}$

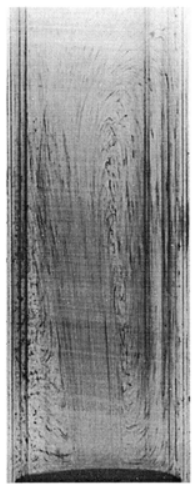

(e) $50 \mathrm{~s}$

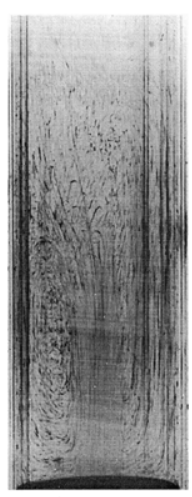

(b) $20 \mathrm{~s}$

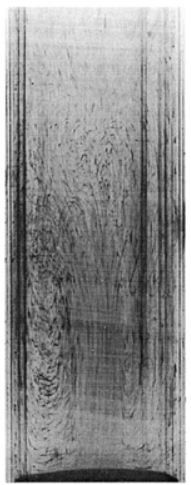

(f) $60 \mathrm{~s}$

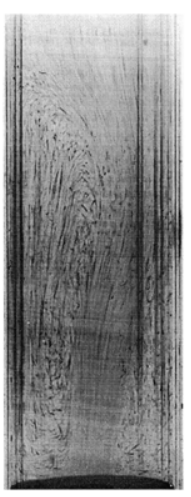

(c) $30 \mathrm{~s}$

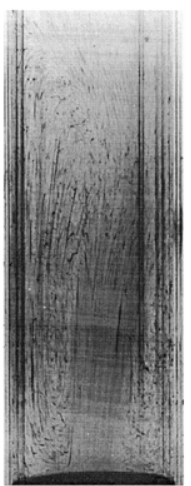

(g) $70 \mathrm{~s}$

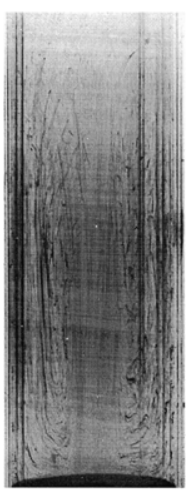

(d) $40 \mathrm{~s}$

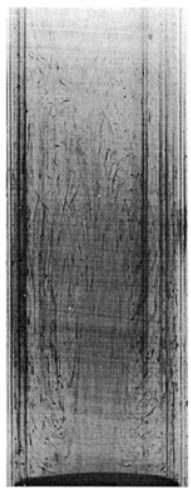

(h) $80 \mathrm{~s}$
Fig. 11. Consecutive photographs of particle paths for the $0.1 \mathrm{wt} \%$ aqueous solution of PAA through an abrupt 4:1 circular contraction at $R e=135, W e=2.48$ and $25^{\circ} \mathrm{C}$ in the globally unstable regime.
Furthermore, Fig. 10 shows that the vortex size on the left hand side remains a little larger during $200 \mathrm{~s}$ at $W e=2.07$, thus this flow regime is not global instability but fully local instability stage. Finally, Fig. 11 exhibits typical unstable flow $(W e=2.48)$ : alternate growth and decay phenomenon of the both side vortices occur: this phenomenon is almost periodic. Furthermore, we show the non-dimensional vortex length $L_{v}^{*}=L_{v} / 2 R_{u} v s$. We from steady flow to globally unstable flow regime in Fig. 12, where $L_{v}$ is the salient corner vortex length and $R_{u}$ is the radius of the upstream tube. In this figure a circle and a square marks are plotted when the length of right hand side corner vortex and left hand side one are different. The circle mark expresses the maximum vortex length and the square one the minimum vortex length, respectively. Furthermore, we show the approximate Weissenberg number of the commencement of local instability, fully local instability and globally unstable flow in Fig. 12. The upper part of the larger salient corner vortex expands up to the centerline of the circular tube in the globally unstable flow regime (see Fig. 11), thus it is clearly found from Fig. 12 that the vortex length in the global instability becomes smaller than the vortex length at $W e=2.07$ in the fully local instability flow regime. The vortex lengths on the both sides become a little different at $W e=2.07$, however, the upper part of the larger vortex does not expand toward the centerline of the tube (see Fig. 10).

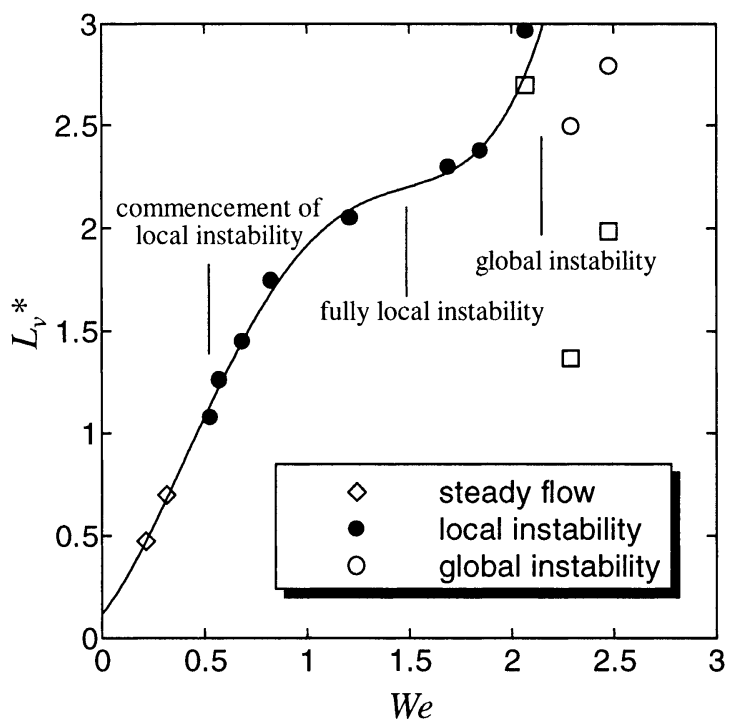

Fig. 12. Non-dimensional vortex length vs. Weissenberg number from steady flow to globally unstable flow regime for the $0.1 \mathrm{wt} \%$ aqueous solution of PAA through an abrupt $4: 1$ circular contraction at $25^{\circ} \mathrm{C}$. 


\section{FLOW STRUCTURE NEAR THE DETACHMENT POINT AND ALONG THE VORTEX BOUNDARY}

Flow visualization experiments near the tube wall were conducted in order to confirm the occurrence of small amplitude oscillation of the detachment point in the local instability regime. When the detachment point oscillates, the dye-solution should diffuse. The dye-solution was injected along the tube wall and the diffusion of the dye-solution near the detachment point was observed.

It is confirmed that streakline does not change with time for steady flow. And it is also difficult to detect the change in the streakline with time for the initial stage of local instability in the vortex region ( $W e=0.743$, Fig. 13).

When $W e$ is further increased to $W e=0.954$, interesting flow patterns can be observed in the visualization using the dyesolution injection technique. The consecutive photographs of Fig. 14 clearly show the occurrence of a small dip of the vortex

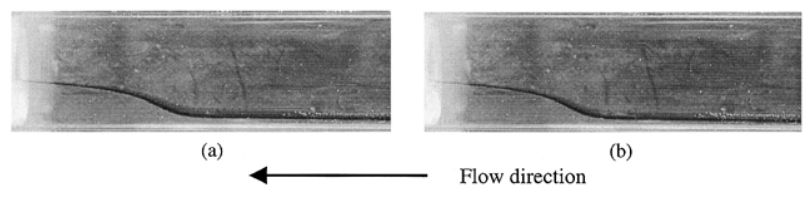

Fig. 13. Streakline photographs for the $0.1 \mathrm{wt} \%$ aqueous solution of PAA through an abrupt 4:1 circular contraction at $R e=1.60, W e=0.743$ and $25^{\circ} \mathrm{C}$ at the initial stage of local instability.

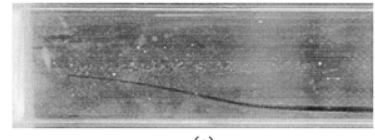

(a)

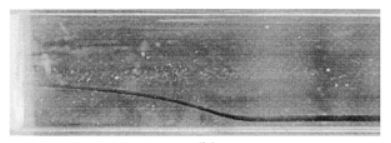

(b)

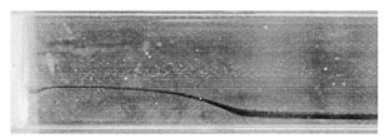

(c)

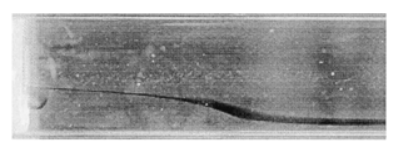

(d)

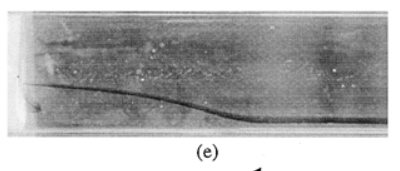

(e)

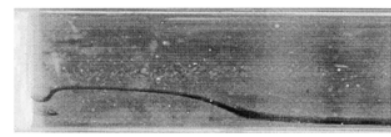

(f)

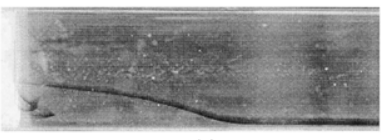

(g)

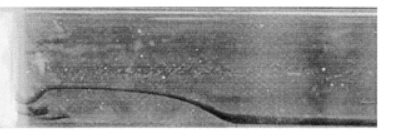

(h)

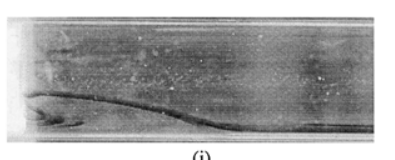

(i)

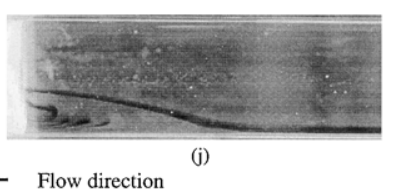

Fig. 14. Consecutive photographs of streakline for the $0.1 \mathrm{wt} \%$ aqueous solution of PAA through an abrupt 4:1 circular contraction at $R e=4.03, W e=0.954$ and $25^{\circ} \mathrm{C}$ in the local instability regime. boundary at the lip corner in Fig. 14(c), 14(f) and 14(h), and also the occurrence and decay of the dip are almost periodically repeated. Furthermore, the dye-solution in the dip can flow into the salient corner vortex at the moment of the decay of the dip (Fig. 14(d), 14(g) and 14(i)). We can also confirm that the expansion and shrinkage of the vortex boundary are exhibited synchronously to the occurrence and decay of the dip of the vortex boundary. This complex phenomenon is definitely twodimensional. We can easily understand that non-smooth particle paths in the vortex observed in the tracer-particle visualizations arise from the expansion and shrinkage phenomenon of the vortex boundary owing to the occurrence and decay of the dip at the lip corner. Similar flow patterns can be seen in the flow visualization results for creeping flow through a $4: 1$ circular contraction of Boger fluid $(0.04 \mathrm{wt} \%$ PAA, Separan AP30, in corn syrup and water solution) conducted by Boger et al. (Fig. 11) ${ }^{17)}$, however, they did give no comment about the occurrence of a dip of the vortex boundary at the lip corner. Furthermore, Rothstein and McKinley (Fig. 17) ${ }^{18)}$ first reported a high-speed 'jetting' instability near the lip corner in a 4:1:4 circular contractionexpansion of $0.025 \mathrm{wt} \%$ PS/PS Boger fluid, which was photographed and measured using the tracer-particle visualization and DPIV technique: a strong jet of high speed fluid appears, originating from the base of the precessing axisymmetric upstream vortex at the point of its greatest upstream extent and flowing directly into the contraction. A brief video image can be also seen in their website. They, however, did not recognize the above synchronous expansion and shrinkage of the salient corner vortex.

Flow around the vortex becomes further complicated as $W e$ increases. In Fig. $15(W e=1.31)$ a larger dip of the vortex boundary periodically occurs at the lip corner and the expansion and shrinkage of the vortex boundary are repeated. In addition, the streakline gradually becomes thick around the detachment point (Fig. 15(b) and 15(i)), then the thick streakline separates at the moment of the decay of the dip (Fig. 15(c) and 15(j)). In Fig. 15(c) the upper streakline connects to the lower one in the region drawn by ellipse between these two streaklines and also the separation point of the upper streakline from the tube wall abruptly moves upstream in comparison with that of Fig. 15(b). Then the upper streakline approaches the lower one and the separation length gradually becomes shorter in Fig. 15(c) $(\mathrm{h})$ (from $L_{s}^{*}=L_{s} / 2 R_{u}$ to 2.30). Finally coincidence of the upper and lower streaklines can be attained in Fig. 15(h). In this complex case, however, the flow remains to be two-dimensional. 
Furthermore, at $W e=1.82$, not the global instability but the fully local instability regime, the streakline exhibits spiraling curve in the vortex region (Fig. 16(d) (j)), i.e., the vortex flow becomes three-dimensional. These flow patterns could not be

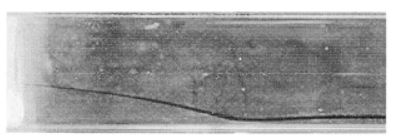

(a)

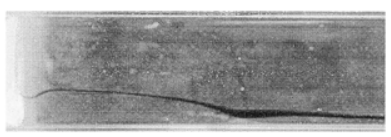

(b)

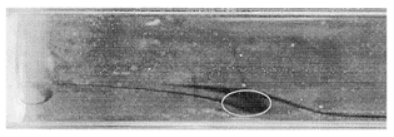

(c)

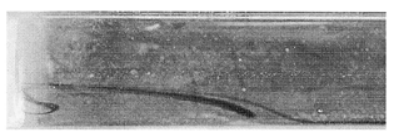

(d)

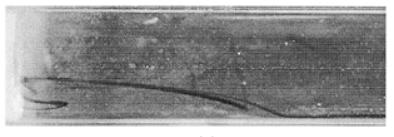

(e)

$\leftarrow$

Fig. 15. Consecutive photographs of streakline for the $0.1 \mathrm{wt} \%$ aqueous solution of PAA through an abrupt 4:1 circular contraction at $R e=13.0, W e=1.31$ and $25^{\circ} \mathrm{C}$ in the local instability regime.

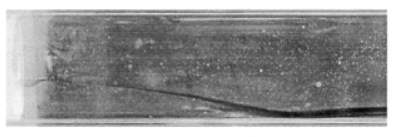

(a)

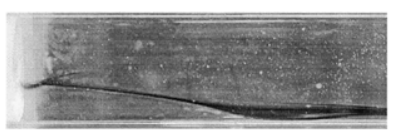

(b)

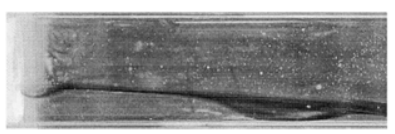

(c)

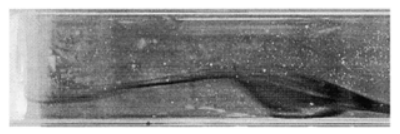

(d)

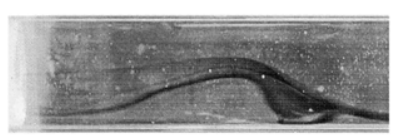

(e)

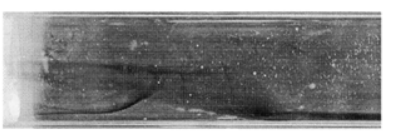

(f)

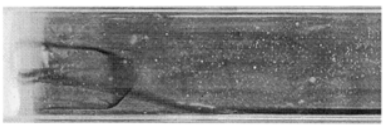

(g)

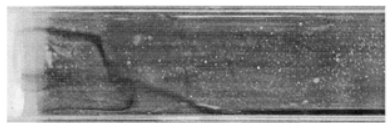

(h)

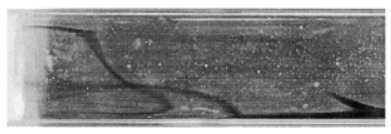

(i)

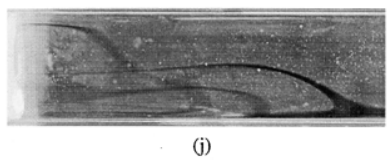

Flow direction
Fig. 16. Consecutive photographs of streakline for the $0.1 \mathrm{wt} \%$ aqueous solution of PAA through an abrupt 4:1 circular contraction at $R e=43.3, W e=1.82$ and $25^{\circ} \mathrm{C}$ in the fully local instability regime. recorded by the photographs taken in the central plane of the tube in the visualization experiments using the tracer method, e.g., Fig. 10. Thus the above results demonstrate that the dyesolution injection technique is very useful to detect whether a flow is two- or three-dimensional. Finally, the distortion of the streakline can be clearly observed in the globally unstable flow using the dye-solution injection technique as well.

In consequence, change in the vortex shape arises mainly from the occurrence and decay of a dip of the vortex boundary at the lip corner, as a result, non-smooth particle paths can be observed in the vortex flow and near the vortex boundary. The small amplitude oscillation of the detachment point due to the interaction between inertial force and elastic force described by $\mathrm{Naka}^{6}{ }^{\text {) }}$ has, therefore, a secondary influence on the local instability phenomenon, i.e., visible non-smooth particle paths near the detachment point is ascribable to the small amplitude oscillation of the detachment point at the commencement of local instability $(W e \approx 0.5)$.

\section{CONCLUDING REMARKS}

In contraction flow, extensional properties dominantly encourage vortex growth in steady vortex growing regime. However, the conflict between fluid inertia and elasticity has a dominant influence on the behavior of fully grown salient corner vortex in the region near the detachment point: the equilibrium state between inertial force and elastic force can be destroyed owing to small disturbance of flow or nonhomogeneity of fluid. $\mathrm{Naka}^{6}$ ) first postulated from the visualization experiments using the tracer method that local instability, which appears as non-smooth particle paths, in the salient corner vortex arises from the oscillatory phenomenon of the detachment point. A curved streamline and tensile stress along the streamline are necessary for the elastic instability to occur as proposed by McKinley et al.. ${ }^{13)}$ In the vicinity of the detachment point, streamlines are curved and the polymer molecules are stretched along the streamlines.

However, in the present study flow visualizations using the dye-solution injection technique demonstrated that the main cause of the local instability in the vortex region is the occurrence and decay of a dip of the vortex boundary at the lip corner, while the commencement of visible non-smooth particle paths near the detachment point arises from the small amplitude oscillatory phenomenon of the detachment point. Local instability in the vortex region can evolve to the global instability of entry flow as the Weissenberg number increases. We have, therefore, confirmed that the occurrence of a dip of 
the vortex boundary at the lip corner is a trigger of the global entry flow instability after vortex enhancement. However, the mechanism of the occurrence of a dip of the vortex boundary at the lip corner and its arrangement in the circular direction remains to be an open question.

At the next step in the frame work of the local instability phenomenon of entry flow, it will be necessary to carry out the quantitative measurement of the velocity field around the lip corner in both the vortex and main flow regions using the LDV or PIV technique to fully understand not only the mechanism of the occurrence of a dip of the vortex boundary at the lip corner but also complex flow kinematics due to stress singularity around the lip corner.

\section{Acknowledgements}

The authors are grateful to Dr. Y. Naka for his kind permission in reproducing photographs in Figs.1, 2, and also the reviewer for the useful and significant suggestions.

\section{REFERENCES}

1) Cable PJ, Boger DV, AIChE J, 25, 152 (1979).

2) Nguyen H, Boger DV, J Non-Newtonian Fluid Mech, 5, 353 (1979).

3) Cartalos U, Piau JM, J Non-Newtonian Fluid Mech, 45, 231 (1992).
4) Piau JM, El Kissi N, Tremblay B, J Non-Newtonian Fluid Mech, 30, 197 (1988).

5) Boger DV, Walters K, "Rheological Phenomena in Focus", (1993), Rheology series, Elsevier, Amsterdam.

6) Naka Y, "Flow kinematics of polymer solution through an abrupt contraction", (1998), Ph.D thesis, Osaka University.

7) Lawler JV, Muller SJ, Brown RA, Armstrong RC, J NonNewtonian Fluid Mech, 20, 51 (1986).

8) McKinley GH, Raiford WP, Brown RA, Armstrong RC, $J$ Fluid Mech, 223, 411 (1991).

9) Chiba K, Nakamura K, J Non-Newtonian Fluid Mech, 73, 67 (1997).

10) Naka Y, Chiba K, Nakamura K, Nihon Reoroji Gakkaishi (J Soc Rheol, Jpn), 26, 41 (1998).

11) Naka Y, Chiba K, Nakamura K, Trans(B) Japan Soc Mech Eng, 64, 2019 (1998).

12) Shaqfeh ESG, Ann Rev Fluid Mech, 28, 129 (1996).

13) McKinley GH, Pakdel P, Öztekin A, J Non-Newtonian Fluid Mech, 67, 19 (1996).

14) Tanner RI, “Engineering Rheology”, (1988), Oxford University Press, New York.

15) Barnes HA, Hutton FJ, Walters K, "Introduction to Rheology", (1989), Elsevier, Amsterdam.

16) Binding DM, Maia J, Walters K, J Non-Newtonian Fluid Mech, 52, 137 (1994).

17) Boger DV, Hur DU, Binnington RJ, J Non-Newtonian Fluid Mech, 20, 31 (1986).

18) Rothstein JP, McKinley GH, J Non-Newtonian Fluid Mech, 98, 33 (2001). 